\title{
Evaluation of shoulder internal rotator muscle strength in baseball players with physical signs of glenohumeral joint instability
}

\author{
D L Falla, S Hess, C Richardson
}

See end of article for authors' affiliations

....................

Correspondence to: Ms Falla, Department of Physiotherapy, University of Queensland, St Lucia, Brisbane, Queensland 4072, Australia;

d.falla@shrs.uq.edu.au

Accepted

2 October 2002
Objectives: To identify whether subjects with glenohumeral translational instability present with a strength deficit in their internal rotator musculature, and therefore to determine if general strengthening exercises are warranted for the management of this condition.

Methods: Eighteen male baseball players were allocated to two groups on the basis of expert clinical evaluation of the presence $(n=8)$ or absence $(n=10)$ of shoulder translational instability. A strain gauge was used to calculate the force generated during maximal isometric internal rotation contractions in neutral shoulder rotation and $30^{\circ}$ of internal rotation.

Results: The Mann-Whitney test identified no significant difference in force production during the maximum contractions in neutral rotation between the two subject groups. In the position of $30^{\circ}$ shoulder internal rotation, subjects with glenohumeral translational instability produced significantly greater force than the control group $(p<0.05)$.

Conclusions: High load strength testing cannot be used as a diagnostic tool to identify people with glenohumeral instability. Similarly, heavy resistance strengthening exercises for the internal rotator musculature are not warranted during rehabilitation.
S houlder instability is a significant problem for many athletes. Therefore the development of effective conservative management is essential. There seems, however, to be no consensus on the most appropriate form of exercise management during rehabilitation of shoulder instability.

On the basis of muscle deficits considered to occur in shoulder instability, two types of formal exercise programmes have been used to improve stability of the shoulder joint. These two types of exercise are focused on two different functional muscle groups. Muscles can be defined as "local" or "global" according to their functional roles. ${ }^{1}$ Local muscles include the vastus medialis obliqus of the knee, the transversus abdominis and multifidus of the lumbar spine, and, in the case of the glenohumeral joint, the rotator cuff. These muscles are associated with joint stability. The local rotator cuff muscles are recruited during a presetting phase before shoulder movement to enhance stability of the glenohumeral joint. ${ }^{2}$ The larger global muscles, such as latissimus dorsi and pectoralis major of the shoulder, are characterised by originating a considerable distance from the underlying joints. ${ }^{3}$ This, combined with the fact that these muscles are usually composed of long fibres, renders them less capable of providing stability to the joints that they influence. ${ }^{3}$

General strengthening exercises are commonly prescribed for rehabilitation..$^{4-7}$ These exercises involve higher resistance training and thus recruit all the muscle synergists - that is, both the local and global muscles. For example, strengthening the internal rotators of the shoulder would recruit all muscles that can contribute to internal rotation: subscapularis, teres major, pectoralis major/minor, and latissimus dorsi. Another type of exercise programme has been designed to focus on the dynamic stabilisers. ${ }^{89}$ These exercises aim to improve control of the deep rotator cuff muscles in their role as stabilisers of the glenohumeral joint-for example, subscapularis and teres major during internal rotation. General strengthening exercises are not recommended in this exercise approach, as it is considered that the large global muscles become overactive, presumably to compensate for dysfunction in the deep muscles. ${ }^{10}{ }^{11}$ General strengthening exercises would probably exacerbate this dysfunction.

As the two exercise approaches used to treat joint dysfunction are opposite with regard to strength training, a study was designed in the first instance to assess if subjects with glenohumeral translational instability present with a strength deficit. Baseball players were chosen because the incidence of shoulder pathologies is high in this population. It was important to choose subjects who had translational instability but no pain so that this variable could be eliminated. Internal rotation was selected because a strong and effective concentric contraction of the shoulder internal rotator muscles is required during throwing.

To identify whether subjects with glenohumeral translational instability present with a strength deficit in their internal rotator musculature, the average force produced during maximum voluntary contractions of isometric shoulder internal rotation in both neutral rotation and $30^{\circ}$ of internal rotation was compared between subjects with glenohumeral translational instability and normal matched controls.

\section{METHODS}

\section{Subjects}

Eighteen male baseball players, aged between 17 and 32 years, participated. Subjects were allocated to two groups based on expert clinical evaluation of shoulder translational instability in the dominant arm. A physiotherapist, skilled in assessing shoulder stability, performed the anterior draw test ${ }^{12}$ on subjects during the initial recruitment session in order to assign them to a group. All subjects were chosen to closely represent each other in terms of age, weight, and height.

Subjects with shoulder translational instability $(\mathrm{n}=8)$ were aged between 18 and 32 years (mean (SD) 25.7 (4.9)). Shoulder translational instability was defined as an anterior draw test range greater in the dominant arm than the non-dominant arm, in association with a different "end feel" and greater shoulder external rotation. ${ }^{13}$ This increased range 


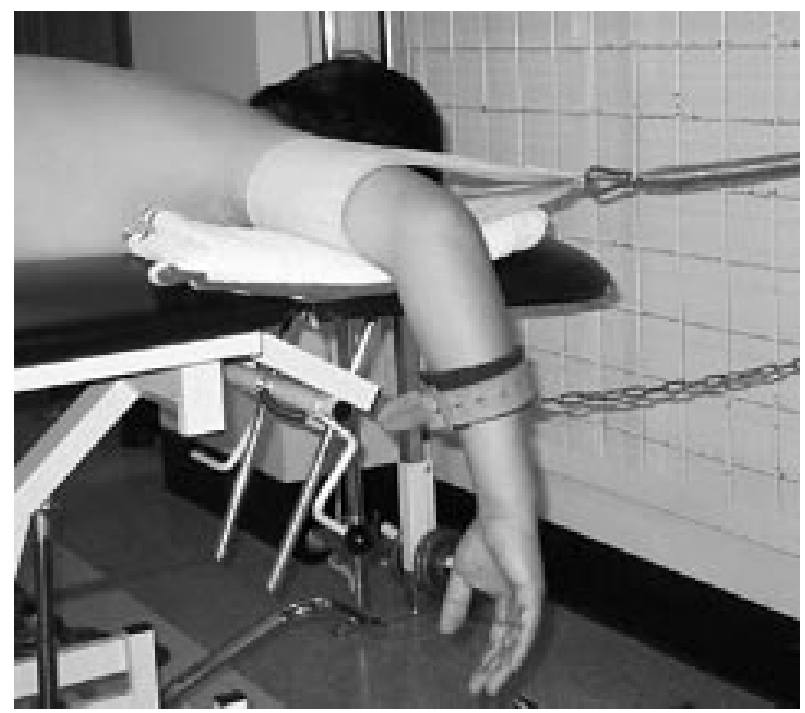

Figure 1 Each subject was positioned on a plinth in the prone position as in the standard internal rotation muscle test. A leather wrist strap was positioned $15 \mathrm{~cm}$ distal to the olecranon process and, via a chain, was securely attached to a strain gauge. To avoid glenohumeral adduction during the testing procedure, the humerus was stabilised using a sling attached to a grid in front.

is considered to signify greater range in the anterior glenohumeral capsule compared with the non-dominant side. Subjects were still playing competitive baseball, were not limited in their physical capacity, and did not complain of any pain in the shoulder region at the time of testing.

Subjects in the control group $(\mathrm{n}=10)$ were aged between 17 and 29 years (mean (SD) 20.8 (3.5)). To be included in this group, subjects had to demonstrate an equal anterior draw test range and normal "end feel" in their dominant and non-dominant arm. To participate in this study, all subjects had to have at least $90^{\circ}$ of shoulder abduction and $30^{\circ}$ of internal rotation. Exclusion criteria were $(a)$ a history of glenohumeral dislocation on the dominant side, $(b)$ pain in the dominant shoulder, cervical spine, or thoracic spine at the time of testing, or (c) any previous shoulder or elbow surgery or previous fracture in the shoulder area on the dominant side.

Written informed consent was obtained from all volunteers, and ethical approval for the study was granted by the medical research ethics committee of the University of Queensland.

\section{Instrumentation and measurements}

A strain gauge was used to calculate the force generated by the shoulder internal rotator muscles during maximal isometric internal rotation contractions. With the subject positioned prone, the strain gauge was securely attached to a frame at one end, and, via a chain, the other end was connected to a leather strap fastened on the subjects forearm (fig 1). The chain was attached to the frame so that it formed a right angle with the forearm. The position of the wrist strap was standardised by positioning the proximal edge of the strap $15 \mathrm{~cm}$ distal to the olecranon process.

An AMLAB data acquisition system was used to collect the force data. The data collection software was designed to display the maximum force generated at the completion of each maximum contraction.

The force generated during maximum isometric shoulder internal rotation contractions was measured in two test positions, $0^{\circ}$ and $30^{\circ}$ of shoulder internal rotation, to represent two ranges of muscle length during the strength test. Both test positions required the subject to lie prone with their shoulder at $90^{\circ}$ of abduction as in the standard muscle test for the internal rotators. ${ }^{14}$ A goniometer was used to determine the
Table 1 Basic details of the subjects

\begin{tabular}{|c|c|c|c|c|c|c|}
\hline \multirow[b]{2}{*}{ Group } & \multicolumn{2}{|c|}{ Age (years) } & \multicolumn{2}{|c|}{ Height $(\mathrm{cm})$} & \multicolumn{2}{|c|}{ Weight (kg) } \\
\hline & Mean & SD & Mean & SD & Mean & SD \\
\hline $\begin{array}{l}\text { Controls } \\
\text { Instability }\end{array}$ & $\begin{array}{l}20.8 \\
25.75\end{array}$ & $\begin{array}{l}3.52 \\
4.95\end{array}$ & $\begin{array}{l}181.80 \\
184.63\end{array}$ & $\begin{array}{l}6.36 \\
6.80\end{array}$ & $\begin{array}{l}75.20 \\
81.63\end{array}$ & $\begin{array}{l}13.03 \\
9.55\end{array}$ \\
\hline
\end{tabular}

Table 2 Results of the Mann-Whitney test for differences in the maximum force produced in subjects with and without shoulder translational instability (Trans Inst)

\begin{tabular}{ll}
\hline Joint position & $\mathrm{p}$ Value \\
\hline Neutral rotation & 0.2303 \\
Thirty degrees shoulder internal rotation & \\
$\quad$ Trans Inst $\neq$ controls & 0.0832 \\
$\quad$ Trans Inst > controls & $0.0416^{*}$ \\
\hline *Significant result. & \\
\hline
\end{tabular}

position of $30^{\circ}$ of shoulder internal rotation. The fulcrum of the goniometer was positioned over the olecranon process with the stationary arm of the goniometer perpendicular to the floor. The ulnar styloid process was then used as a reference point to align the movable arm. ${ }^{14}$

Visual analogue scales were used to monitor pain levels during the contractions, and, if a subject recorded pain greater than a scale of 1 throughout testing, the results were not included. No subjects recorded pain during the testing procedures.

\section{Experimental procedure}

The testing environment was optimum to increase the subject's ability to produce maximum contractions. This included an air conditioned room with minimal distractions.

Subjects were positioned on a plinth in the prone position as in the standard internal rotation muscle test ${ }^{14}$ (fig 1 ). Passive shoulder internal rotation was used to indicate the direction of pull required by the subjects. The strain gauge was then attached to the subject's forearm by a chain. To avoid glenohumeral adduction during the testing procedure, the humerus was stabilised using a sling attached to a grid in front.

Once comfortably positioned, the subject practised an isometric shoulder internal rotation contraction, and corrections of the subject's performance were made as necessary. Subjects then received verbal encouragement while performing two maximum voluntary isometric internal rotation contractions in the neutral rotation position with the dominant arm. The subject's shoulder was then repositioned in $30^{\circ}$ of internal rotation using a goniometer. The bed height and position of the strain gauge was adjusted to ensure that a $90^{\circ}$ angle was maintained between the strain gauge and forearm. A further two maximum isometric internal rotation contractions were performed. Each contraction was sustained for five seconds. An interval of five minutes was allowed between each repetition. The highest value of force recorded over the two maximum contractions became the maximum voluntary contraction for each test position.

\section{Data management}

The Mann-Whitney test was used to identify whether significant differences existed between the two subject groups for the maximum force produced in each test position. The Mann-Whitney test was also used to assess any difference in the amount of force produced between the two test positions. $\mathrm{p}<0.05$ was considered significant. 
Table 3 Results of the Mann-Whitney test for differences in the amount of force produced in neutral shoulder rotation and $30^{\circ}$ of shoulder internal rotation

\begin{tabular}{ll}
\hline Subject group & $\mathrm{p}$ Value \\
\hline Controls & $0.0023 *$ \\
Translational instability & $0.0330^{*}$ \\
\hline *Significant result. & \\
\hline
\end{tabular}

\section{RESULTS}

Table 1 gives the basic details of the subjects studied.

No significant difference was found between the amount of force produced during the maximum contraction in the neutral rotation position for the two subject groups. In the position of $30^{\circ}$ shoulder internal rotation, subjects with glenohumeral translational instability produced significantly greater force than the control group $(\mathrm{p}<0.05)$ (table 2$)$.

Significantly greater force was produced in the neutral joint position than at $30^{\circ}$ internal rotation. This difference was identified for both subject groups (table 3 ).

\section{DISCUSSION}

The results indicate that subjects with glenohumeral translational instability do not have an isometric strength deficit in their internal rotator musculature. Subjects with glenohumeral translational instability showed equal strength to controls in the neutral rotation position, and, when tested in $30^{\circ}$ of shoulder internal rotation, they showed greater force output than the controls. The results must be interpreted with caution, however, because the validity and repeatability of the anterior draw test to differentiate between subjects with and without glenohumeral translational instability was not examined in this study.

These results support previous findings of studies of the lumbar and cervical spine which showed that, in the presence of joint dysfunction, there is not necessarily a loss of strength. ${ }^{15} 16$ There is evidence from these musculoskeletal conditions that muscle dysfunction occurs in the deep muscles. ${ }^{10}{ }^{11}$ In relation to the glenohumeral joint, it has been hypothesised that deficits probably occur in the deep rotator cuff muscles and not the larger muscles such as the pectoralis major and latissimus dorsi. Therefore, the results of this study, as well as findings from previous research on other joints, suggest that strengthening exercises using high resistance may not be the optimal exercise regimen for joint instability.

Future research on shoulder translational instability should concentrate on investigating the presence of dysfunction in the deep rotator cuff of the shoulder and assessing the nature of the dysfunction. Once this has been established, therapeu-
Take home message

- Strength testing cannot be used as a diagnostic tool to identify people with glenohumeral translational instability

- Strengthening exercises using high resistance may not be the optimal exercise regimen for joint instability

tic exercise that will enhance glenohumeral stability can be developed.

\section{Authors' affiliations}

D L Falla, S Hess, C Richardson, Department of Physiotherapy,

University of Queensland, Brisbane, Queensland, Australia

\section{REFERENCES}

1 Bergmark A. Stability of the lumbar spine. A study in mechanical engineering. Acta Orthop Scand Suppl 1989;60(suppl 230): 1-54.

2 David G, Magarey ME, Jones MA, et al. EMG and strength correlates of selected shoulder muscles during rotations of the glenohumeral joint. Clin Biomech 2000;15:95-102.

3 Williams PL, Bannister LH, eds. Gray's anatomy. 38th ed. Edinburgh: Churchill Livingstone, 1995.

4 Bradley J, Tibone J. Elctromyographic analysis of muscle action about the shoulder. Clin Sports Med 1991;10:789-805.

5 Burkhead WZ, Rockwood CA. Treatment of instability of the shoulder with an exercise program. J Bone Joint Surg [Am] 1992;74:890-6.

6 Townsend $\mathbf{H}$, Jobe FW, Pink M, et al. Electromyographic analysis of the glenohumeral muscles during a baseball rehabilitation program. Am J Sports Med 1991;19:264-72.

7 Wilk KE, Arrigo C. Current concepts in the rehabilitation of the athlete shoulder. J Orthop Sports Phys Ther 1993;18:365-75

8 Dalton S, Snyder S. Glenohumeral instability. In: Bailliere's clinical rheumatology. Philadelphia: Balliere Tindall, 1989:511-33.

9 Kamkar A, Irrgang J, Whitney S. Nonoperative management of secondary shoulder impingement syndrome. J Orthop Sports Phys Ther 1993; 17:212-24

10 Jull GA. Deep cervical flexor muscle dysfunction in whiplash. Journal of Musculoskeletal Pain 2000:8:143-54.

11 Ng JK-F, Ricardson C, Parnianpour M, et al. Fatigue related changes in torque output and electromyographic parameters in trunk muscles during isometric axial rotation exertion: an investigation in patients with back pain and in healthy subjects. Spine 2002;27:637-46.

12 Magee D. Orthopaedic physical assessment. Philadelphia: WB Saunders, 1997.

13 Hertling D, Kessler RM. Management of common musculoskeletal disorders: physical therapy principles and methods. 3rd ed. Philadelphia: Lippincott, 1996.

14 Clarkson HM, Gilewich GB. Musculoskeletal assessment: joint range of motion and manual muscle strength. Sydney: Williams and Wilkins, 1989.

15 Falla D, Rainoldi A, Merletti R, et al. Myoelectric manifestations of sternocleidomastoid and anterior scalene muscle fatigue in chronic neck pain patients. XIVth Congress of the International Society of

Electrophysiology and Kinesiology, Vienna, Austria, June 22-25, 2002 Clin Neurophysiol 2003;1 14:488-95.

16 Ng JK-F, Richardson CA, Parnianpour M, et al. EMG activity of trunk muscles and torque output during isometric axial rotation exertion: a comparison between back pain patients and matched controls. J Orthop Res 2002;20: $112-21$ 